Louisiana State University

LSU Digital Commons

$9-1-2004$

\title{
Macrocycle-derived functional xanthenes and progress towards concurrent detection of glucose and fructose
}

\author{
Oleksandr Rusin \\ Louisiana State University \\ Onur Alpturk \\ Louisiana State University \\ Ming $\mathrm{He}$ \\ Louisiana State University \\ Jorge O. Escobedo \\ Louisiana State University \\ Shan Jiang \\ Louisiana State University
}

See next page for additional authors

Follow this and additional works at: https://digitalcommons.Isu.edu/chemistry_pubs

\section{Recommended Citation}

Rusin, O., Alpturk, O., He, M., Escobedo, J., Jiang, S., Dawan, F., Lian, K., McCarroll, M., Warner, I., \& Strongin, R. (2004). Macrocycle-derived functional xanthenes and progress towards concurrent detection of glucose and fructose. Journal of Fluorescence, 14 (5), 611-615. https://doi.org/10.1023/ B:JOFL.0000039348.74270.03

This Article is brought to you for free and open access by the Department of Chemistry at LSU Digital Commons. It has been accepted for inclusion in Faculty Publications by an authorized administrator of LSU Digital Commons. For more information, please contact ir@lsu.edu. 
Authors

Oleksandr Rusin, Onur Alpturk, Ming He, Jorge O. Escobedo, Shan Jiang, Fareed Dawan, Kun Lian, Matthew E. McCarroll, Isiah M. Warner, and Robert M. Strongin 


\title{
Macrocycle-Derived Functional Xanthenes and Progress Towards Concurrent Detection of Glucose and Fructose
}

\author{
Oleksandr Rusin ${ }^{1,2}$, Onur Alpturk ${ }^{1}$, Ming He ${ }^{1,3}$, Jorge O. Escobedo ${ }^{1}$, Shan Jiang ${ }^{1}$, Fareed \\ Dawan $^{2}$, Kun Lian ${ }^{2}$, Matthew E. McCarrolI ${ }^{1,4}$, Isiah M. Warner ${ }^{1}$, and Robert M. Strongin ${ }^{1,5}$ \\ ${ }^{1}$ Department of Chemistry, Louisiana State University, Baton Rouge, Louisiana 70803 \\ ${ }^{2}$ Center for Advanced Microstructures and Devices, Louisiana State University, Baton Rouge, \\ Louisiana
}

\begin{abstract}
The detection of saccharides in biological media is of great current importance for the monitoring of disease states. We have previously reported that solutions of boronic acid-functionalized macrocycles form acyclic oligomeric materials in situ. The oligomers contain fluorescent xanthene moieties. Current efforts are aimed at modulating the spectroscopic responses of these materials for the analysis of specific sugars. We describe conditions whereby the xanthene boronic acids exhibit high colorimetric fructose selectivity. In contrast, at physiological levels selective glucose monitoring can be achieved via fluorescence. Additionally, we describe a method which exhibits promise for detecting both glucose and fructose at dual wavelengths in the UV-Vis region. Mechanistic rationale for each of these findings is presented.
\end{abstract}

\section{Keywords}

Glucose; fructose; resorcinarene; boronic acid; xanthene

\section{INTRODUCTION}

There has been great progress made towards the design, synthesis and evaluation of organic dyes functionalized with boronic acids for sugar detection [1]. There are relatively few studies, however, addressing the simultaneous detection of common sugars, such as glucose and fructose, in mixtures [1,2]. It has been known for decades that boronic acids exhibit relatively high affinity for fructose compared to other saccharides [3]. More recently several glucose-selective chemosensors have been synthesized [1]. Nearly all are based on the key discovery by Shinkai and co-workers that scaffolds containing appropriately spaced bisboronic acid moieties may selectively chelate glucose [1]. Herein we describe novel methodology which shows promise for the detection of both glucose and fructose via a combination of UV-Vis and fluorescence spectroscopy.

Previously we reported the facile synthesis and isolation of $\mathbf{1}$ on multi-gram scale via the acid-catalyzed condensation of resorcinol and 4-formyphenylboronic acid [4]. Compound 1 is soluble in aqueous polar aprotic solvents such as DMSO. We observed that colorless DMSO solutions containing 1 (Fig. 1), upon standing or heating at $90^{\circ} \mathrm{C}$ for $1 \mathrm{~min}$, turned

\footnotetext{
(C) 2004 Springer Science+Business Media, Inc.

${ }_{3}^{5}$ To whom correspondence should be addressed. rstrong@1su.edu.

3 Present address: Advanced Biotech, Inc., Patterson, New Jersey.

${ }^{4}$ Present address: Department of Chemistry and Biochemistry, Southern Illinois University, Carbondale, Illinois.
} 
pinkish-purple. These color changes were monitored with UV-Vis spectroscopy via the appearance of new absorptions at 465, 500 and $536 \mathrm{~nm}$ [5].

We found that upon heating eleven aqueous DMSO solutions each containing boronic acid resorcinarene macrocycles and eleven respective saccharides, a different solution color could be observed by visual inspection corresponding to each sugar [5]. The colorimetric responses were rapid, quantifiable and reproducible. More recently, we applied similar methodology towards the colorimetric detection of neutral oligosaccharides [6].

Mechanistic investigations, based on extensive spectroscopic, chromatographic and crystallographic studies, revealed that the color formation in the macrocycle-containing solutions was due to the formation of ring-opened acyclic oligomers possessing xanthene chromophores (2, Fig. 1) [6]. Our studies were greatly facilitated by the pioneering work of Weinelt and Schneider who had earlier described the reversible mechanism of resorcinarene macrocycle genesis in homogeneous solutions [7].

We determined that the sugar-promoted signal transduction arose via the formation of anionic sugar-boronate esters [6], in keeping with the well-known properties of boronic acid-functionalized dyes [1]. This was confirmed by ${ }^{13} \mathrm{C}$ NMR using isotopically labeled sugars [6]. Our results were consistent with the important and useful NMR-based characterizations of related sugar-arylboronates performed by Norrild and co-workers [8].

\section{EXPERIMENTAL}

Reagents, solvents and human blood plasma were purchased from Sigma-Aldrich. Lyophilized blood plasma $(5.0 \mathrm{~mL})$ was reconstituted with $\mathrm{H}_{2} \mathrm{O}(2.0 \mathrm{~mL})$ and deproteinized by addition of $\mathrm{MeCN}(3.0 \mathrm{~mL})$. Clear filtrate was used for the glucose detection experiments.

All spectroscopic data was acquired at room temperature. Colored solutions of $\mathbf{1}$ were produced via preheating DMSO solutions and cooling to room temperature prior to adding water or plasma and analytes. UV-Vis data was obtained using a Spectramax Plus 384 spectrophotometer (Molecular Devices). Fluorescence spectra were recorded with a HR2000 fiber optic spectrometer (Ocean Optics). The excitation wavelength was $470 \mathrm{~nm}$ (Ocean Optics LS-450, blue LED) directed into a fluorescence cuvette via a $600 \mu \mathrm{m}$ entrance fiber, and emission gathered by a $1000 \mu \mathrm{m}$ optical fiber at $\lambda_{\max } 579 \mathrm{~nm}$. Configured for fluorescence, the setup used two mirrored screw plugs positioned at $90^{\circ}$ within the cuvette holder for signal enhancement.

\section{RESULTS AND DISCUSSION}

\section{Selective Detection of Fructose and the Ratiometric Monitoring of Fructose and Glucose Via UV-Vis Spectroscopy}

We find that fructose promotes a striking solution color change in the presence of preheated, colored aryl-boronic acid resorcinarene $\mathbf{1}$ (from pink-purple) instantly at room temperature. Colorless chemosensor $1(5.2 \mathrm{mM})$ is heated at a gentle reflux for $3 \mathrm{~min}$ in DMSO $(0.9 \mathrm{~mL})$ in air to afford a colored solution containing 1 . After cooling to room temperature, fructose (1 equiv) in $0.1 \mathrm{~mL} \mathrm{H}_{2} \mathrm{O}$ is added. A color change from pink-purple to orange-yellow is observed. Glucose, sucrose, maltose, lactose and xylose, 3 equiv each, exhibit no color change within $2 \mathrm{hr}$ at room temperature (Fig. 2).

The color change is monitored by observing the ratiometric absorbance intensity decrease at $536 \mathrm{~nm}$ and increase at $464 \mathrm{~nm}$ (Fig. 3). The absorbance changes exhibit a linear 
dependence with fructose concentration $(R=0.9079$ and 0.9419 at each of the two wavelengths, respectively).

The ratio of glucose to fructose in blood plasma is ca. 100:1. Addition of 1 equivalent of fructose to a colored solution containing 1 at room temperature results in an $8.6 \%$ increase in the absorbance at $464 \mathrm{~nm}$. Addition of 100 equivalents of glucose to the fructose/1 solution results in no detectable change in the fructose/1 absorbance at $464 \mathrm{~nm}$. Addition of a second equiv of fructose to this latter solution results in a readily observable absorbance increase of $3.3 \%$.

At $536 \mathrm{~nm}$, the absorbance of solutions of $\mathbf{1}$ is lowered by $20 \%$ upon addition of 1 equiv fructose. Subsequent addition of 100 equivalents of glucose lowers the absorbance further by $12 \%$. Addition of another equivalent of fructose again lowers the absorbance by $24 \%$ (Fig. 3).

This result indicates the potential feasibility of determining fructose, in the presence of excess of glucose, by monitoring fructose concentrations at $464 \mathrm{~nm}$, where an absorbance change is not produced in response to glucose. In addition, one should be able to concurrently determine the glucose present in a sample via analysis of the ratio of the absorbance at $536 \mathrm{~nm}$ (at which wavelength both glucose and fructose promote signal changes) to the absorbance at $464 \mathrm{~nm}$, for instance, after the fructose concentration is determined at $464 \mathrm{~nm}$.

The selectivity for fructose appears consistent with our previous binding constant studies of neutral sugars [6]. Fructose binds monoboronic acids with a greater affinity than glucose due to a favorable configuration of hydroxyls [1]. Additionally, we had determined that anionic fructoseboronate formation lowers the $\mathrm{p} K_{\mathrm{a}}$ of the colored xanthenes (2), resulting in absorbance changes [6]. It is well-known that xanthene dyes show an increase in absorbance at their shorter visible wavelength and a decrease in absorbance at their longer visible wavelength in response to a lowering of solution $\mathrm{pH}$. Thus, we can ascribe the ratiometric responses observed at 464 and $536 \mathrm{~nm}$ in the current studies as due, in large part, to a lowering of xanthene $\mathrm{p} K_{\mathrm{a}}$ upon sugar binding.

The UV-Vis studies described above would require higher concentrations of sugars than typically found in most naturally-occurring biological samples in order to generate useful signals. We are investigating the synthesis and study of new functional xanthene dyes with enhanced sensitivity in order to avoid concentration steps in sample monitoring. Since the xanthenes (2) are present in colored solutions only at micromolar levels [6], their synthesis and/or isolation as discreet compounds should afford materials with higher colorimetric sensitivity, since a significant amount of sugar binds to excess colorless boronic acid $\mathbf{1}$. They (2) are attained readily via the highly facile synthesis of $\mathbf{1}$ [6]. The presence of an excess of visually inactive binding sites appears to diminish sensitivity; however, high fructose selectivity is attained. The proportion of boronic acid binding sites functioning as signal induction-promoting moieties can also favorably influence selectivity in these systems in fluorescence experiments, as described below. Colored solutions containing 1 show promise for monitoring glucose levels in the range of physiological levels via fluorescence spectroscopy.

\section{Enhanced Glucose Selectivity Via Fluorescence Detection in the Range of Physiological Concentrations and in Human Blood Plasma}

The fluorescence emission spectra of colored solutions containing 1 and added glucose or fructose are shown in Fig. 4. As the sugar concentration is increased we observe concomitant emission increases promoted by fructose and glucose. The significant signaling 
generated by glucose is in contrast to the UV-Vis studies (vide supra) in which relatively much weaker absorbance responses were promoted by glucose as compared to fructose.

The normal level of D-fructose in human blood plasma is ca. $50 \mu \mathrm{M}$. Fluorescence emission spectra of colored solutions containing 1 and added fructose, obtained over the fructose concentration range of 20 to $100 \mu \mathrm{M}$, exhibit no detectable fructose-promoted emission. Healthy levels of D-glucose are ca. $5 \mathrm{mM}$. The emission spectra of glucose $\left(5.5 \times 10^{-3} \mathrm{M}\right)$ and $\mathbf{1}\left(1.0 \times 10^{-3} \mathrm{M}\right)$ in DMSO: $\mathrm{H}_{2} \mathrm{O}, 9: 1$, as well as in a 9:1 DMSO:plasma solution, are shown in Fig. 5. Emission increases due to the presence of glucose are observed in both cases. A dependence of fluorescence emission intensity on increased glucose levels in plasma is observed (Fig. 5).

The results of the fluorescence studies show that the boronic acid-functionalized xanthenes (2) may promote the detection of glucose in blood plasma with negligible interference from fructose. New UV-Vis and near-IR absorbing congeners of $\mathbf{2}$, possessing functionality for greater potential glucose signaling ability, are currently being designed in our lab to address potential fluorescence interference issues in blood plasma.

The enhanced fluorescence emission promoted by glucose, as compared to the its relatively weaker UV-Vis responses (e.g., relative to fructose), may be attributed to chelation by neighboring boronic acids of $\mathbf{2}$. It is well-known that glucose can be chelated by bis-boronic acids. This results in chemosensor scaffold rigidification effects, which have been previously demonstrated to afford fluorescence emission enhancement [1,9]. An excess of boronic acid binding sites relative to glucose should promote chelation. The large excess of non-absorbing 1 compared to responsive fluorophores (2) should thus facilitate glucosepromoted emission by competing for glucose binding to 2 .

\section{CONCLUSION}

We have presented evidence that oligomeric xanthene dye-functionalized boronic acids, which form in situ from tetraarylboronic acid resorcinarene macrocycles, show promise for the selective detection of glucose and fructose. The fluorescence studies indicate that selectivity for glucose over fructose at physiological levels in plasma may be achieved. Via UV-Vis spectroscopy, we observe high fructose selectivity. Additionally, ratiometry may also be used to simultaneously measure fructose and glucose levels, at proportionally higher concentrations. The mechanistic insights gained from these results will aid us in designing improved xanthene-derived chemosensors with tuneable properties. The synthesis of water soluble and surface-bound congeners of the molecules described herein is also in progress.

\section{Acknowledgments}

We are very grateful to the National Institutes of Health for supporting this research via grant 8R01EB002044-03.

\section{References}

1. James TD, Shinkai S. Artificial receptors as chemosensors for carbohydrates. Top Curr Chem. 2002; 218:159-200.(b) Wang W, Gao X, Wang B. Boronic acid-based sensors. Curr Org Chem. 2002; 6(14):1285-1317.

2. Arimori S, Bell ML, Oh CS, Farimat KA, James TD. Modular fluorescence sensors for saccharides. Chem Commun. 2001:1836-1837.

3. Lorand JP, Edwards JD. Polyol complexes and structure of the benzeneboronate ion. J Org Chem. $1959 ; 24: 769-774$. 
4. Lewis PT, Davis CJ, Saraiva M, Treleaven D, McCarley T, Strongin RM. Tetraarylboronic acid resorcinarene stereoisomers. Versatile new substrates for divergent polyfunctionalization and molecular recognition. J Org Chem. 1997; 62(18):6110-6111.

5. Davis CJ, Lewis PT, McCarroll ME, Read MW, Cueto R, Strongin RM. Simple and rapid visual sensing of saccharides. Org Lett. 1999; 1(2):331-334. [PubMed: 10905872]

6. He M, Johnson RJ, Escobedo JO, Beck PA, Kim KK, St Luce NN, Davis CJ, Lewis PT, Fronczek FR, Melancon BJ, Mrse AA, Treleaven WD, Strongin RM. Chromophore formation in resorcinarene solutions and the visual detection of mono- and oligosaccharides. J Am Chem Soc. 2002; 124(18):5000-5009. [PubMed: 11982364]

7. Weinelt F, Schneider H-J. Mechanisms of macrocycle genesis. The condensation or resorcinol with aldehydes. J Org Chem. 1991; 56(19):5527-5535.

8. Norrild JC, Eggert H. Boronic acids as fructose sensors. Structure determination of the complexes involved using (1)J(CC) coupling constants. J Chem Soc, Perkin Trans. 1996; 2(12):2583-2588.

9. (a) Takeuchi M, Yoda S, Imada T, Shinkai S. Chiral sugar recognition by a diboronic-acid-appended binaphtyl derivative through rigidification effect. Tetrahedron. 1997; 53(25):8335-8348.(b)

Takeuchi M, Mizuno T, Shinmori H, Nakashima M, Shinkai S. Fluorescence and CD spectroscopic sugar sensing by a cyanine-appended diboronic acid probe. Tetrahedron. 1996; 52(4):1195-1204. 


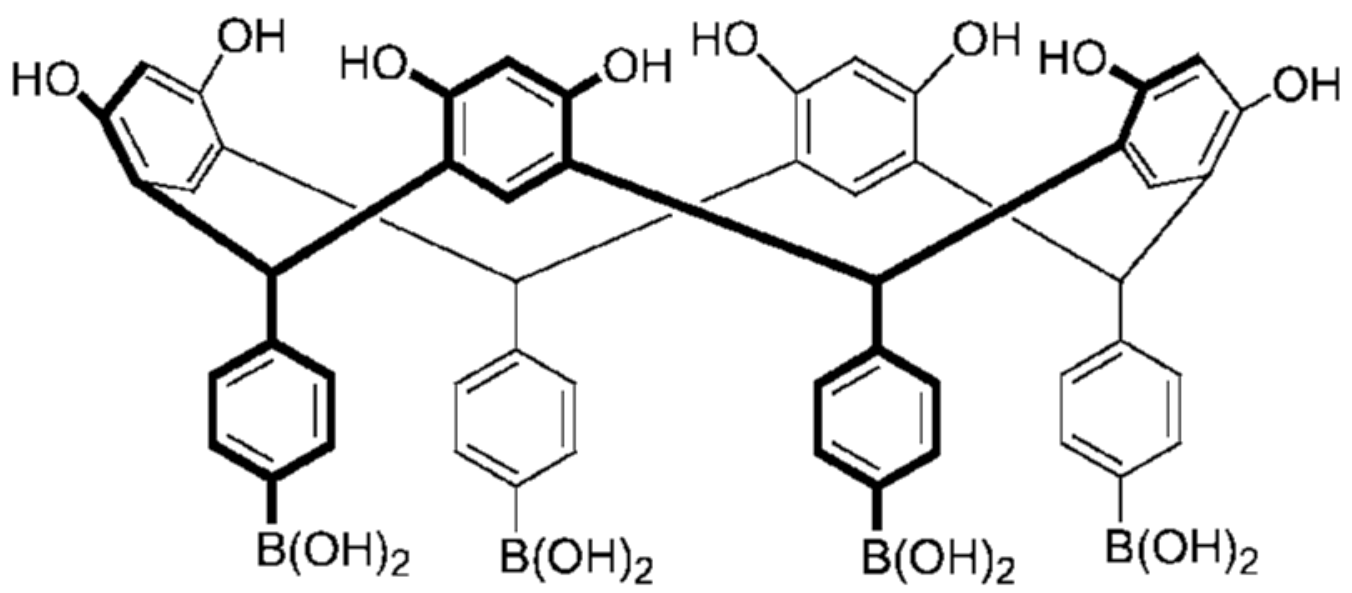

$-\mathrm{H}_{2} \mathrm{O}$<smiles>CC(c1ccc([SeH])cc1)(c1ccc([SeH])cc1)c1cc2c(-c3ccc(O)cc3)c3cc(O)c(=O)cc-3oc2cc1C(c1ccc([SeH])cc1)c1ccc([SeH])cc1</smiles>

2

$$
\begin{aligned}
& n=0,1,2, \text { etc. } \\
& m=0,1,2 \text {, etc. }
\end{aligned}
$$

Fig. 1.

Ring opening of resorcinarene boronic acid macrocycle $\mathbf{1}$ affords acyclic oligomers containing xanthene moieties. 


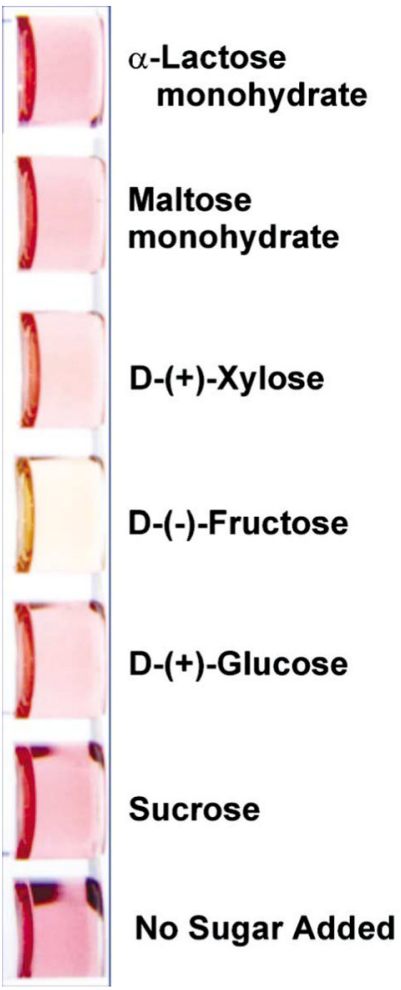

Fig. 2.

A selective color change promoted by fructose is observed at room temperature upon addition to a colored solution containing $\mathbf{1}$. 

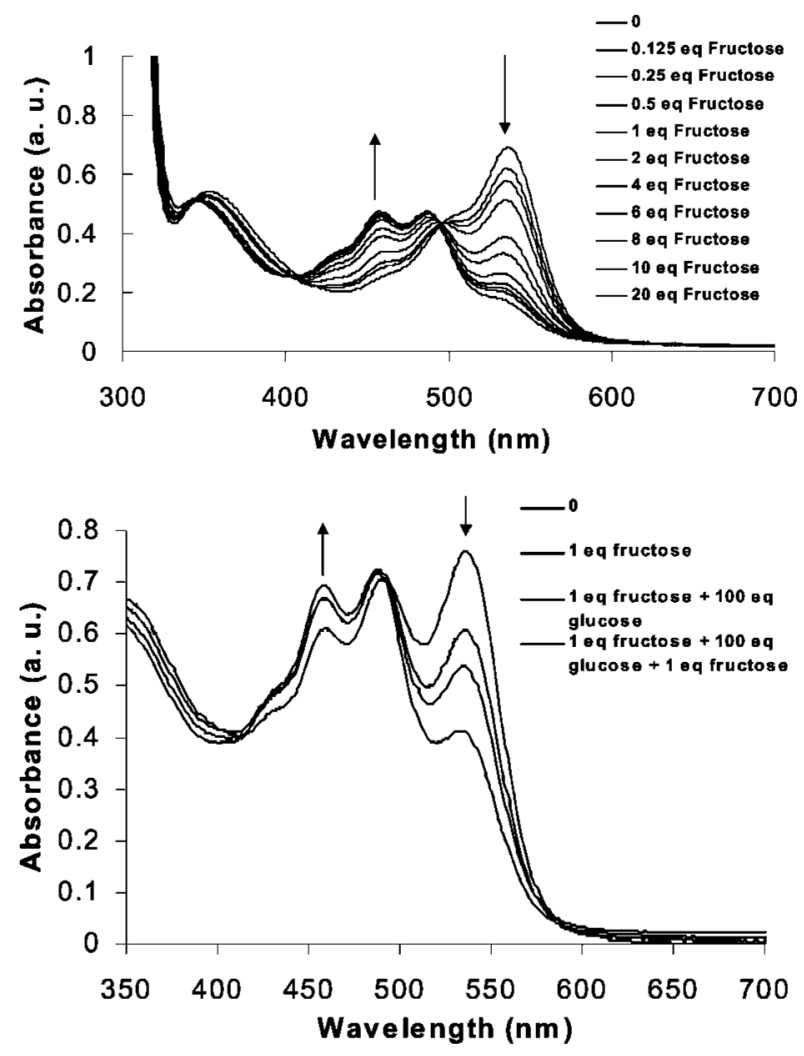

Fig. 3.

Upper: Addition of fructose to a preheated (3.0 min at reflux) solution of $1\left(5.2 \times 10^{-3} \mathrm{M}\right)$ in DMSO at room temperature affords concentration-dependent absorbance changes at 464 and $536 \mathrm{~nm}$. Lower: UV-Vis spectra of a 9:1 DMSO: $\mathrm{H}_{2} \mathrm{O}$ solution containing $1\left(5.2 \times 10^{-3} \mathrm{M}\right)$ pre-heated (1.5 min at reflux) (i) alone, (ii) upon addition of 1 equiv fructose at room temperature which produces an absorbance increase at $464 \mathrm{~nm}$ and a corresponding decrease at $536 \mathrm{~nm}$, (iii) upon addition of 100 equiv glucose which produces no absorbance change at $464 \mathrm{~nm}$ but a decrease at $536 \mathrm{~nm}$ and (iv) upon addition of a second equivalent of fructose which affords a further absorbance increase at $464 \mathrm{~nm}$ and decrease at $536 \mathrm{~nm}$. 


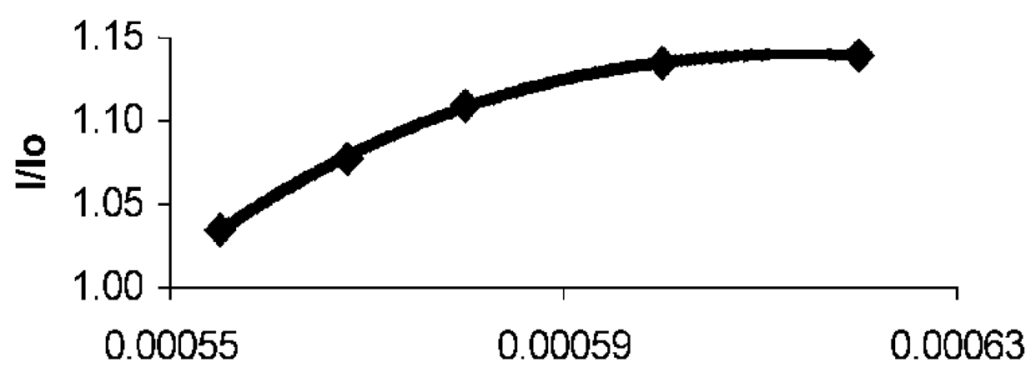

[D-glucose] M

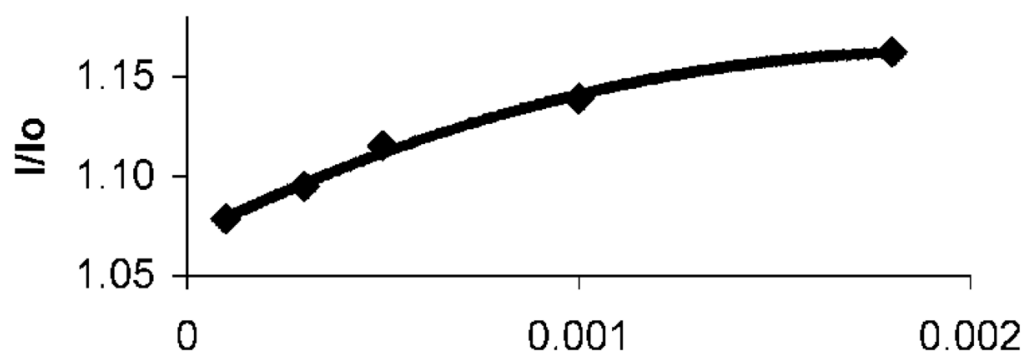

[D-fructose] $\mathrm{M}$

Fig. 4.

Upper: Fluorescence emission changes produced upon addition of D-glucose to a colored solution (DMSO: $\left.\mathrm{H}_{2} \mathrm{O} 9: 1\right)$ containing $1\left(5.0 \times 10^{-3} \mathrm{M}\right)$ at room temperature. The glucose concentration was increased from 0 to $6.2 \times 10^{-4} \mathrm{M}$. Lower: Fluorescence emission changes produced upon addition of D-fructose to a colored solution (DMSO: $\mathrm{H}_{2} \mathrm{O}$ 9:1) containing 1 $\left(5.0 \times 10^{-3} \mathrm{M}\right)$ at room temperature. The fructose concentration is increased from 0 to $1.8 \times$ $10^{-3} \mathrm{M}$. The fluorescence intensity increases (corresponding to the increase of glucose or fructose concentration) are 13.9 and $18.2 \%$, respectively. The emission changes are obtained at glucose levels that are smaller than those of fructose. 

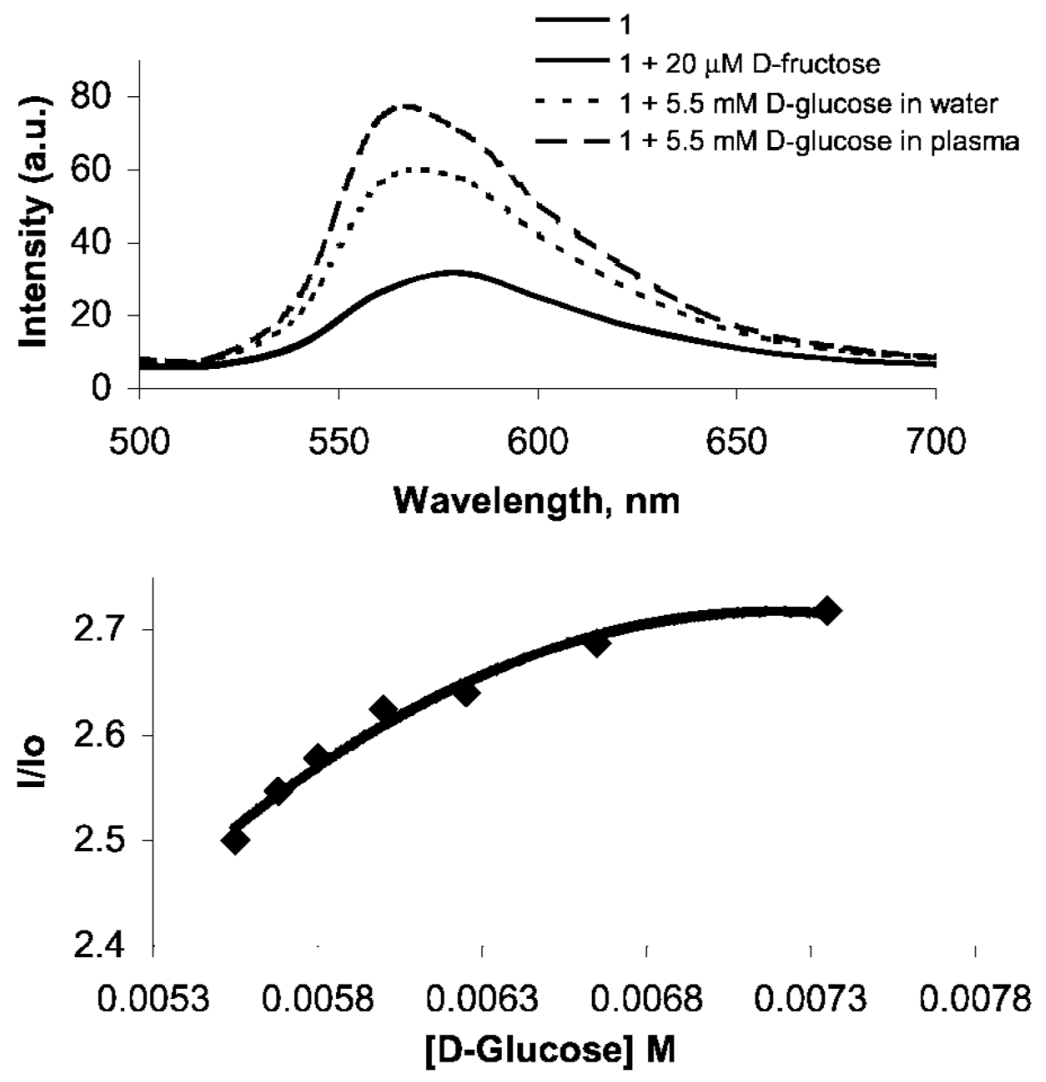

Fig. 5.

Upper: Fluorescence emission spectra produced by (i) a preheated ( $3 \mathrm{~min}$ at reflux) colored solution (DMSO: $\left.\mathrm{H}_{2} \mathrm{O} 9: 1\right)$ containing $1\left(5.0 \times 10^{-3} \mathrm{M}\right)$ at room temperature, (ii) the same conditions but in the presence of $20 \mu \mathrm{M}$ D-fructose, added at room temperature, which affords no observable change in emission, (iii) the same conditions as (i) but with added Dglucose $(5.3 \mu \mathrm{M})$ which promotes an emission increase and (iv) same conditions as (iii) but in deproteinized human blood plasma instead of $\mathrm{H}_{2} \mathrm{O}$, which exhibits an emission increase in response to added glucose. Lower: Concentration-dependent emission changes produced via room temperature additions of D-glucose to a 9:1 DMSO:plasma solution containing 1. 